\title{
Pneumococcal glomerulonephritis in a healthy child: a case report and literature review
}

\author{
Intan Hakimah Ismail ${ }^{1}$, MD, MMed, Zurina Zainudin ${ }^{1}$, MBBS, MRCPCH, Norlijah Othman ${ }^{1}$, MBBs, MRCP
}

\begin{abstract}
Pneumococcal glomerulonephritis is rarely described in the literature. We report a four-year-old boy who developed acute glomerulonephritis following pneumococcal bacteraemia and submandibular lymphadenitis, and review the published literature. Two weeks after developing acute glomerulonephritis, the patient developed bronchopneumonia with left pleural effusion. However, by the fourth week of admission, his renal function had normalised and lung involvement resolved.
\end{abstract}

Keywords: glomerulonephritis, pleural effusion, pneumonia, Streptococcus pneumoniae

\section{INTRODUCTION}

Nephritogenic strains of group A $\beta$-haemolytic streptococci (GABHS), such as Streptococcus (S.) pyogenes, are the most common cause of post-infectious acute glomerulonephritis (AGN) following throat or skin infections. Isolated reports of acute nephritis caused by $S$. pneumoniae infection, usually following respiratory symptoms, have rarely been described. ${ }^{(1,2)}$ Herein, we present a previously healthy child who developed pneumococcal AGN following submandibular and cervical lymphadenitis complicated with respiratory problems, and in whom AGN was confirmed on blood culture. We also review the relevant literature.

\section{CASE REPORT}

A previously healthy four-year-old boy presented to Hospital Kuala Lumpur, Malaysia, with a one-week history of fever and neck swelling. Two days prior to admission, he developed high fever, vomiting and diarrhoea, with poor oral intake and reduced urine output. On examination, the boy was lethargic, febrile (temperature at $39.9^{\circ} \mathrm{C}$ ) and appeared ill. His pulse rate was $120 \mathrm{bpm}$, respiratory rate was $30 \mathrm{bpm}$ and blood pressure was 98/46 $\mathrm{mmHg}$ (50th percentile for age, gender and height at $93 / 50 \mathrm{mmHg}$ ). The patient was noted to have bad dental caries, cracked lips and dry mucous membrane with otherwise warm peripheries and normal capillary refilling time. There were enlarged, tender and non-fluctuant left submandibular $(3 \mathrm{~cm} \times$ $3 \mathrm{~cm})$ and submental $(2 \mathrm{~cm} \times 1 \mathrm{~cm})$ lymph nodes. He also had multiple bilateral cervical lympha-denopathies and a 3-cm hepatomegaly. Physical examination was otherwise unremarkable. Past medical and family histories were insignificant, and immunisation was complete (Malaysia's routine immunisation programme does not include heptavalent pneumococcal conjugate vaccine).

Laboratory investigations showed elevated white blood cells (WBCs; $\left.28.8 \times 10^{9} / \mathrm{L}\right)$ with neutrophilia (93.7\%). Blood urea nitrogen $(21.4 \mathrm{mmol} / \mathrm{L})$ and serum creatinine $(138 \mu \mathrm{mol} / \mathrm{L})$ were also elevated. The other blood parameters investigated were haemoglobin $(8.4 \mathrm{~g} / \mathrm{dL})$, platelets $\left(399 \times 10^{9} / \mathrm{L}\right)$, C-reactive protein $(5.20 \mathrm{mg} / \mathrm{dL})$ and serum albumin $(24 \mathrm{~g} / \mathrm{L}$; normal range 35-50 g/L). Serum complement C3 was at $0.21 \mathrm{~g} / \mathrm{L}$ (normal range $0.55-1.20 \mathrm{~g} / \mathrm{L}$ ), while $\mathrm{C} 4$ was at $0.23 \mathrm{~g} / \mathrm{L}$ (normal range, $0.20-0.50 \mathrm{~g} / \mathrm{L}$ ). Urinalysis and microscopic examination revealed $3+$ proteins, numerous red blood cells (RBCs) and WBCs, with 2+ RBCs, 2+ WBCs and 2+ granular casts per highpowered field. Spot urine protein/creatinine index was 0.21 and 24-hour urine protein was at $25 \mathrm{mg} / \mathrm{m}^{2} /$ hour. Results of the patient's liver function test were normal. Antistreptolysin $\mathrm{O}$ titre (ASOT) was elevated at $800 \mathrm{IU} / \mathrm{mL}$ (normal range, $<200 \mathrm{IU} / \mathrm{mL}$ ). Quantitative immunoglobulins were normal and connective tissue studies were negative.

Initial chest radiography on admission was normal. The first blood culture grew $S$. pneumoniae sensitive to penicillin and cephalosporin. However, further tests for pneumococcal serotyping and minimal inhibitory concentration (MIC) were unavailable. Cultures from the patient's throat swab and urine were sterile. Renal ultrasonography demonstrated bilateral enlarged kidneys with increased echogenicity.

The patient's initial management was focused on the possible causes of cervical lymphadenitis. Intravenous (IV) penicillin (50,000 unit/kg/dose) and cefotaxime (50 mg/kg/dose) were commenced. Over the following 48 hours, the patient developed periorbital and leg oedema, with tea-coloured urine. The patient was subsequently treated for acute nephritis with fluid restriction, and put on a low-salt diet and close monitoring of daily fluid intake/urine output. His blood pressure remained stable throughout the course of hospitalisation.

Despite being administered antibiotics, the patient's fever remained unresolved. After two weeks of hospitalisation, he developed bronchopneumonia with left pleural effusion that was confirmed on repeat chest radiography. A left pleural tap did not grow any organisms, but the effusion did not deteriorate.

${ }^{1}$ Department of Paediatrics, Universiti Putra Malaysia, Selangor, Malaysia

Correspondence: Dr Intan Hakimah Ismail, Paediatrician and Medical Lecturer, Department of Paediatrics, Faculty of Medicine and Health Sciences, Universiti Putra Malaysia, 43400 UPM Serdang, Selangor, Malaysia. ihakimah@yahoo.com 
Table I. Clinical and epidemiological data of patients reported to have pneumococcal glomerulonephritis in the literature.

\begin{tabular}{|c|c|c|c|c|c|c|c|c|c|}
\hline $\begin{array}{l}\text { Study, } \\
\text { year }\end{array}$ & $\begin{array}{l}\text { Age of } \\
\text { patient } \\
\text { (yrs)/ } \\
\text { gender/ } \\
\text { country }\end{array}$ & $\begin{array}{l}\text { Nature of } \\
\text { primary } \\
\text { infection }\end{array}$ & $\begin{array}{l}\text { Interval } \\
\text { between } \\
\text { primary } \\
\text { infection } \\
\text { and renal } \\
\text { symptoms }\end{array}$ & $\begin{array}{l}\text { Renal abnormalities } \\
\text { on admission }\end{array}$ & $\begin{array}{l}\text { Blood } \\
\text { parameters }\end{array}$ & $\begin{array}{l}\text { Chest } \\
\text { radiography } \\
\text { findings }\end{array}$ & $\begin{array}{l}\text { Culture } \\
\text { results }\end{array}$ & Treatment & Outcome \\
\hline $\begin{array}{l}\text { Hyman } \\
\text { et al, } \\
1975^{(4)}\end{array}$ & $\begin{array}{l}4 / F / \\
\text { USA }\end{array}$ & $\begin{array}{l}\text { Lower } \\
\text { respiratory } \\
\text { tract infection }\end{array}$ & 2 days & $\begin{array}{l}\text { Haematuria, } \\
\text { proteinuria } \\
(300 \mathrm{mg} / \mathrm{dL}) \text {, pyuria, } \\
\text { BP }(90 / 60 \mathrm{mmHg})\end{array}$ & $\begin{array}{l}\text { BUN: } 15 \mathrm{mmol} / \mathrm{L} \\
\text { SCr: } 0.7 \mathrm{mg} / \mathrm{dL} \\
\text { C3/C4: } \\
\text { reduced/normal } \\
\text { ASOT: elevated }\end{array}$ & $\begin{array}{l}\text { Right } \\
\text { middle lobe } \\
\text { pneumonia }\end{array}$ & $\begin{array}{l}\text { Blood: } \\
\text { S. pneumoniae } \\
\text { serotype } 14 \\
\text { Throat and } \\
\text { urine: negative }\end{array}$ & $\begin{array}{l}\text { Ampicillin } \\
\text { (duration: NA) }\end{array}$ & $\begin{array}{l}\text { Discharged on } \\
\text { Day 5; normal } \\
\text { renal function } \\
\text { and complement } \\
\text { levels at } 8 \text { wks; } \\
\text { normal urinalysis } \\
\text { at } 7-8 \text { mths }\end{array}$ \\
\hline $\begin{array}{l}\text { Kaehny } \\
\text { et al, } \\
1978^{(6)}\end{array}$ & $\begin{array}{l}40 / M / \\
\text { USA }\end{array}$ & $\begin{array}{l}\text { Bacteraemic } \\
\text { left lower lobe } \\
\text { pneumonia }\end{array}$ & 9 days & $\begin{array}{l}\text { Haematuria, } \\
\text { azotaemia, oliguria }\end{array}$ & $\begin{array}{l}\text { BUN: NA } \\
\text { SCr: } 2.4 \mathrm{mg} / 100 \mathrm{~mL} \\
\text { C3/C4: } \\
\text { reduced/reduced } \\
\text { ASOT: NA }\end{array}$ & $\begin{array}{l}\text { Left lower } \\
\text { lobe } \\
\text { pneumonia; } \\
\text { alveolitis and } \\
\text { interstitial } \\
\text { infiltrates at } \\
2 \text { wks }\end{array}$ & $\begin{array}{l}\text { Blood: } \\
\text { S. pneumoniae } \\
\text { serotype } 9\end{array}$ & $\begin{array}{l}\text { Penicillin G, } \\
\text { prednisone } \\
\times 7 \text { wks, } \\
\text { azathioprine } \\
\times 3 \text { wks }\end{array}$ & $\begin{array}{l}\text { Discharged at } \\
13 \text { wks with } \\
\text { microscopic } \\
\text { haematuria and } \\
\text { proteinuria; } \\
\text { asymptomatic } \\
\text { after } 8 \text { mths }\end{array}$ \\
\hline $\begin{array}{l}\text { Schacter } \\
\text { et al, } \\
1987^{(2)}\end{array}$ & $\begin{array}{l}5 / \mathrm{M} / \\
\text { Israel }\end{array}$ & $\begin{array}{l}\text { High fever, } \\
\text { macroscopic } \\
\text { haematuria }\end{array}$ & $24 \mathrm{hrs}$ & $\begin{array}{l}\text { Haematuria, } \\
\text { proteinuria } \\
\left(0.9 \mathrm{~g} / \mathrm{m}^{2} / \text { hour }\right) \\
\text { pyuria, oliguria, BP } \\
(150 / 100 \mathrm{mmHg})\end{array}$ & $\begin{array}{l}\text { BUN: } 104 \mathrm{mmol} / \mathrm{L} \\
\text { SCr: } 1.2 \mathrm{mg} / \mathrm{dL} \\
\text { C3/C4: } \\
\text { reduced/normal } \\
\text { ASOT: normal }\end{array}$ & $\begin{array}{l}\text { Right } \\
\text { lower lobe } \\
\text { pneumonia }\end{array}$ & $\begin{array}{l}\text { Blood: } \\
\text { S. pneumoniae } \\
\text { serotype } 5\end{array}$ & $\begin{array}{l}\text { Ampicillin } \\
\text { (duration: NA) }\end{array}$ & $\begin{array}{l}\text { Normal renal } \\
\text { function at } \\
\text { discharge on Day } \\
11 \text {; microscopic } \\
\text { haematuria } \\
\text { and proteinuria } \\
\text { persisted for } 1 \mathrm{yr}\end{array}$ \\
\hline $\begin{array}{l}\text { Wolach } \\
\text { et al, } \\
1990^{(3)}\end{array}$ & $\begin{array}{l}3.5 / \mathrm{M} / \\
\text { Israel }\end{array}$ & $\begin{array}{l}\text { Left } \\
\text { submandibular } \\
\text { suppurative } \\
\text { adenitis }\end{array}$ & 12 days & $\begin{array}{l}\text { Haematuria, } \\
\text { proteinuria } \\
(60 \mathrm{mg} / \mathrm{dL}) \\
\mathrm{BP}(140 / 95 \mathrm{mmHg})\end{array}$ & $\begin{array}{l}\text { BUN: NA } \\
\text { SCr: } 1.2 \mathrm{mg} / \mathrm{dL} \\
\text { C3/C4: } \\
\text { reduced/reduced } \\
\text { ASOT: normal }\end{array}$ & $\begin{array}{l}\text { Not } \\
\text { assessed }\end{array}$ & $\begin{array}{l}\text { Submandibular } \\
\text { discharge: } \\
\text { S. pneumoniae } \\
\text { serotype } 15 \\
\text { Throat: } \\
\text { negative }\end{array}$ & $\begin{array}{l}\text { Ampicillin; } \\
\text { surgically } \\
\text { drained } \\
\text { submandibular } \\
\text { abscess }\end{array}$ & $\begin{array}{l}\text { Normal renal } \\
\text { function at } 6 \text { wks }\end{array}$ \\
\hline $\begin{array}{l}\text { Lawlor } \\
\text { et al, } \\
1992^{(8)}\end{array}$ & $\begin{array}{l}56 / M / \\
\text { USA }\end{array}$ & $\begin{array}{l}\text { Right lower leg } \\
\text { cellulitis }\end{array}$ & 5 days & $\begin{array}{l}\text { Haematuria, } \\
\text { proteinuria } \\
\text { ( } 585 \mathrm{mg} / 24 \text { hours), } \\
\text { pyuria, BP ( } 128 / 70 \\
\mathrm{mmHg} \text { ) }\end{array}$ & $\begin{array}{l}\text { BUN: } 61 \mathrm{mmol} / \mathrm{L} \\
\text { SCr: } 1.9 \mathrm{mg} / \mathrm{dL} \\
\text { C3/C4: } \\
\text { normal/normal } \\
\text { ASOT: elevated }\end{array}$ & Normal & $\begin{array}{l}\text { Bulla aspirate } \\
\text { and blood: } \\
\text { S. pneumoniae } \\
\text { serotype } 6 \mathrm{~B}\end{array}$ & $\begin{array}{l}\text { Penicillin } \\
\times 3 \text { wks, } \\
\text { daily wound } \\
\text { debridement }\end{array}$ & $\begin{array}{l}\text { Skin grafting; } \\
\text { discharged at } \\
7 \text { wks }\end{array}$ \\
\hline $\begin{array}{l}\text { Phillips } \\
\text { et al, } \\
2005^{(1)}\end{array}$ & $\begin{array}{l}6 / F / \\
\text { USA }\end{array}$ & $\begin{array}{l}\text { Lower } \\
\text { respiratory } \\
\text { tract infection }\end{array}$ & 7 days & $\begin{array}{l}\text { Haematuria, } \\
\text { proteinuria, pyuria, } \\
\text { spot urine protein/ } \\
\text { creatinine ratio } 3.87 \text {, } \\
\text { BP }(125 / 68 \mathrm{mmHg})\end{array}$ & $\begin{array}{l}\text { BUN: } 31 \mathrm{mmol} / \mathrm{L} \\
\mathrm{SCr}: 2.3 \mathrm{mg} / \mathrm{dL} \\
\mathrm{C} 3 / \mathrm{C} 4: \\
\text { reduced/reduced } \\
\text { ASOT: NA }\end{array}$ & $\begin{array}{l}\text { Left lingular } \\
\text { pneumonia }\end{array}$ & $\begin{array}{l}\text { Blood and } \\
\text { urine: } \text { S. } \\
\text { pneumoniae } \\
\text { group } 7\end{array}$ & $\begin{array}{l}\text { Ceftriaxone, } \\
\text { diltiazem, } \\
\text { lasix, renal } \\
\text { diuresis }\end{array}$ & $\begin{array}{l}\text { Normal renal } \\
\text { function at } 8 \text { wks }\end{array}$ \\
\hline $\begin{array}{l}\text { Usmani } \\
\text { et al, } \\
2007^{(7)}\end{array}$ & $\begin{array}{l}\text { Adult/ } \\
\text { M/USA }\end{array}$ & NA & NA & NA & NA & Pneumonia & $\begin{array}{l}\text { Blood: } \\
\text { S. pneumoniae } \\
\text { serotype } 7\end{array}$ & $\begin{array}{l}\text { Ceftriaxone, } \\
\text { diltiazem, } \\
\text { lasix, renal } \\
\text { diuresis } \\
\text { Pulse steroid } \\
\times 5 \text { days and } \\
\text { renal dialysis }\end{array}$ & $\begin{array}{l}\text { Normal renal } \\
\text { function }\end{array}$ \\
\hline $\begin{array}{l}\text { Lechón } \\
\text { et al, } \\
2010^{(5)}\end{array}$ & $\begin{array}{l}4.25 / \mathrm{M} / \\
\text { Spain }\end{array}$ & $\begin{array}{l}\text { Lower } \\
\text { respiratory } \\
\text { tract infection }\end{array}$ & 3 days & $\begin{array}{l}\text { Haematuria, pyuria, } \\
\text { proteinuria } \\
\left.\text { ( } 19.06 \mathrm{mg} / \mathrm{m}^{2} / \text { hour }\right) \text {, } \\
\text { oliguria } \\
\text { (0.73 mL/kg/hour), } \\
\mathrm{BP}(107 / 72 \mathrm{mmHg})\end{array}$ & $\begin{array}{l}\text { BUN: } 74 \mathrm{mmol} / \mathrm{L} \\
\mathrm{SCr}: 1.11 \mathrm{mg} / \mathrm{dL} \\
\mathrm{C} 3 / \mathrm{C} 4: \\
\text { reduced/normal } \\
\text { ASOT: elevated }\end{array}$ & $\begin{array}{l}\text { Left lower } \\
\text { lobe } \\
\text { pneumonia }\end{array}$ & $\begin{array}{l}\text { Blood: } \\
\text { S. pneumoniae } \\
\text { serotype } 17 \mathrm{~F} \\
\text { Throat swab } \\
\text { and } \\
\text { urine: negative }\end{array}$ & $\begin{array}{l}\text { Cefotaxime, } \\
\text { lasix for } \\
\text { oliguria }\end{array}$ & $\begin{array}{l}\text { Normal renal } \\
\text { function on } \\
\text { discharge; } \\
\text { normal C3 and } \\
\text { no proteinuria } \\
\text { at } 7 \text { wks; } \\
\text { microscopic } \\
\text { haematuria } \\
\text { persisted for } \\
5 \text { mths }\end{array}$ \\
\hline $\begin{array}{l}\text { Present } \\
\text { study, } \\
2014\end{array}$ & $\begin{array}{l}\text { 4/ M/ } \\
\text { Malaysia }\end{array}$ & $\begin{array}{l}\text { Left } \\
\text { submandibular } \\
\text { and cervical } \\
\text { adenitis }\end{array}$ & 7 days & $\begin{array}{l}\text { Haematuria, pyuria, } \\
\text { proteinuria } \\
\text { ( } 25 \mathrm{mg} / \mathrm{m}^{2} / \text { hour), } \\
\text { spot urine protein/ } \\
\text { creatinine ratio } 0.21 \text {, } \\
\text { oliguria, } \\
\text { BP ( } 98 / 46 \mathrm{mmHg})\end{array}$ & $\begin{array}{l}\text { BUN: } 21.4 \mathrm{mmol} / \mathrm{L} \\
\text { SCr: } 138 \mu \mathrm{mol} / \mathrm{L} \\
\text { C3/C4: } \\
\text { reduced/normal } \\
\text { ASOT: elevated }\end{array}$ & $\begin{array}{l}\text { Normal on } \\
\text { admission; } \\
\text { pneumonia } \\
\text { and left } \\
\text { pleural } \\
\text { effusion at } \\
2 / 52\end{array}$ & $\begin{array}{l}\text { Blood: } \\
\text { S. pneumoniae; } \\
\text { serotype: NA }\end{array}$ & $\begin{array}{l}\text { Penicillin } \\
\times 4 \text { wks, } \\
\text { cefotaxime } \\
\times 1 \mathrm{wk}\end{array}$ & $\begin{array}{l}\text { Discharged at } \\
4 \text { wks; normal } \\
\text { renal function } \\
\text { at } 6 \text { wks; } \\
\text { pneumonia } \\
\text { and left pleural } \\
\text { effusion resolved } \\
\text { by } 4 \text { wks }\end{array}$ \\
\hline
\end{tabular}


Repeat blood culturing to rule out possible nosocomial pneumonia was negative. After three weeks in the ward, the patient's fever settled and urine output was maintained at $>1 \mathrm{~mL} / \mathrm{kg}$ /hour. IV penicillin was continued for a total of four weeks while cefotaxime was given for seven days.

Four weeks into hospitalisation, the patient's renal function normalised, with complete resolution of pneumonia and pleural effusion on chest radiography. He was discharged well one month after admission and remained in good health with normal renal function at the six-week follow-up. Repeat urinalysis revealed no microscopic haematuria or proteinuria. The moderate recovery of his renal status made renal biopsy unnecessary. Although he was advised further evaluation in view of the possibility of progressive renal disease, the patient subsequently defaulted follow-up, and we were unable to assess his long-term renal outcome and repeat $\mathrm{C} 3$ assessments.

\section{DISCUSSION}

The aetiological role of $S$. pyogenes, a GABHS, in childhood post-infectious AGN is well established.(1) Glomerulonephritis following infection by other microbial agents such as S. pneumoniae has rarely been described. Following a review of the English language literature, the authors found that only eight patients with pneumococcal glomerulonephritis (5 children, 3 adults) had been reported over the past 3-4 decades. ${ }^{(1-8)}$ The clinical and epidemiological characteristics of these eight patients are presented in Table I.

For the patients reported in the literature to have pneumococcal glomerulonephritis, pneumococcal serotypes 5, 6B, $7,9,14,15$ and $17 \mathrm{~F}^{(1-8)}$ have been described as nephritogenic strains. Similar to our patient, pneumococcal bacteraemia was reported for almost all of these other patients, suggesting that the rarity of pneumococcal glomerulonephritis could be attributed to the invasiveness of the pneumococcal infection itself. There was a preponderance of male gender ( 6 males, 2 females) and paediatric patients $(n=5)$ in all the eight aforementioned patients (mean age 11.8 [range 3.5-56] years). All patients, including ours, had microscopic haematuria, proteinuria and/ or oliguria on admission. Most patients developed high blood pressure $^{(1-3,5)}$ on presentation, but only two required diuretics, ${ }^{(1,5)}$ and only one of these patients needed antihypertensive agents. ${ }^{(1)}$ Pneumococcal infections were treated with either ampicillin, penicillin or cephalosporin. The outcome of renal involvement in these patients ranged from self-limiting course to acute renal failure requiring dialysis ${ }^{(1,7)}$ and steroid treatment. ${ }^{(7)}$ Our patient had uneventful acute nephritis and a stable blood pressure throughout hospitalisation.

The average incubation period between GABHS infection and the development of $\mathrm{AGN}$ ranged between 1 and 2 weeks for patients with pharyngitis and 3-6 weeks for those with skin infections. Patients were usually no longer febrile at the onset of AGN. In patients with pneumococcal AGN, the onset of nephritis was seen within $2-10$ days ${ }^{(3,4,6,8)}$ of the primary infection, manifesting as pneumonia,,$^{(1,2,4-7)}$ submandibular suppurative adenitis $^{(3)}$ or leg cellulitis. ${ }^{(8)}$

In our patient, who developed AGN one week after experiencing submandibular and cervical adenitis, the clinical condition improved although the fever remained unabated. This suggests that glomerulonephritis was unlikely to have been caused by a post-GABHS infection, and that other causes of infective AGN might have been involved. However, despite our patient not having any sore throat or skin lesions to suggest GABHS infection, his blood culture on admission grew S. pneumoniae (even though throat swab culture was negative) indicating that the organism was indeed the causative agent.

In most patients with post-infectious glomerulonephritis following pneumococcal pneumonia, ${ }^{(1,2,4,5)}$ respiratory symptoms were the first to appear, followed by renal manifestations. In our patient, however, renal involvement was the first manifestation and pneumonia with pleural effusion only developed later.

Similar to our patient, the elevation of serum ASOT accompanying glomerulonephritis was noted in three other studies of AGN in association with pneumococcal infection. ${ }^{(4,5,8)}$ ASOT is normally known to be associated with group A streptococci infection. ${ }^{(1,4)}$ Immunofluorescence studies have also shown that elevated serum titre of ASOT is associated with the deposition of pneumococcal polysaccharides in the glomeruli.(4)

Studies have suggested that post-streptococcal glomerulonephritis is mediated by the deposition of circulating immune complexes or causative antigens/pathogens in the glomeruli. ${ }^{(4,6)}$ According to immunofluorescence studies, pneumococcal polysaccharide bound to the mesangium and glomerular basement membrane may activate the classical and/or alternative complement pathways leading to the deposition of such compounds and the subsequent development of reversible glomerulonephritis. ${ }^{(4,6)}$ The reduced C3 and/or C4 levels, as reported in these patients, are compatible with classical and/or alternative complement activation.

To summarise, we herein presented the case of a fouryear-old boy who developed AGN following pneumococcal bacteraemia and submandibular lymphadenitis, and highlighted the unusual presentation of $S$. pneumoniae complicated with AGN. To our knowledge, this is the first report of a patient in whom renal manifestations preceded respiratory symptoms in pneumococcal AGN. S. pneumoniae should be considered in the differential diagnosis of children presenting with febrile AGN with and without respiratory involvement.

\section{REFERENCES}

1. Phillips J, Palmer A, Baliga R. Glomerulonephritis associated with acute pneumococcal pneumonia: a case report. Pediatr Nephrol 2005; 20:1494-5

2. Schacter J, Pomeranz A, Berger I, Wolach B. Acute glomerulonephritis secondary to lobar pneumonia. Int J Pediatr Nephrol 1987; 8:211-4.

3. Wolach B, Uziel Y, Berger I, Pomeranz A. Cat-scratch bacillus and Streptococcus penumoniae causing submandibular suppurative 
adenitis and acute glomerulonephritis. Child Nephrol Urol 1990; 10:158-60.

4. Hyman LR, Jenis EH, Hill GS, Zimmerman SW, Burkholder PM. Alternative c3 pathway activation in pneumococcal glomerulonephritis. Am J Med 1975; 58: 810-4.

5. Lechón FC, Espí Mde L, Abal RP, Peiró JL. Acute glomerulonephritis associated with pneumonia: a review of three cases. Pediatr Nephro 2010; 25:161-4
6. Kaehny WD, Ozawa T, Schwarz MI, et al. Acute nephritis and pulmonary alveolitis following pneumococcal pneumonia. Arch Intern Med 1978; 138:806-8.

7. Usmani SZ, Shahid Z, Wheeler D, Nasser K. A rare case of postinfectious glomerulonephritis caused by pneumococcus in an adult patient. J Nephrol 2007; 20:99-102.

8. Lawlor MT, Crowe HM, Quintiliani R. Cellulitis due to Streptococcus pneumoniae: case report and review. Clin Infect Dis 1992; 14:247-50. 